\title{
LONGITUDINAL EMITTANCE MEASUREMENTS IN THE FERMILAB RECYCLER RING *
}

\author{
C.M. Bhat and John P. Marriner \\ FNAL, Batavia, IL 60510, USA
}

\begin{abstract}
The Recycler Ring (RR) is a new $8 \mathrm{GeV}$ antiproton storage ring at Fermilab. Presently, this machine is being commissioned using protons from the Booster. It uses barrier buckets for stacking, un-stacking and storing the beam. At any given time, the RR is capable of storing proton or antiproton beams in multiple segments azimuthally. These segments of the beam may have widely differing longitudinal emittance and beam intensities and bunch lengths. It is highly essential to be able to measure the longitudinal emittance and keep track of the longitudinal dynamics at various stages of the operation of the RR. In this paper, we discuss a few methods of longitudinal emittance measurements in barrier buckets and discuss their merits and demerits.
\end{abstract}

\section{INTRODUCTION}

The Recycler Ring at Fermilab[1] is the world's largest antiproton storage ring built mainly using permanent magnets. For stacking the antiprotons in the RR, the beam will be transferred either from the Fermilab Accumulator Ring (cold beam) or from the Tevatron (unused hot beam) via the Main Injector (MI). The hot and cold beams are stored in the RR in separate regions and cooled further. When beam is needed for the Tevatron collider operation, the cold beam from the RR will be extracted in the form of small bunches and transferred to the $\mathrm{MI}$ and accelerated to $150 \mathrm{GeV}$ before injection into the Tevatron. All of these rf manipulations in RR are carried out using barrier buckets.

During the last year we had several antiproton stores in $\mathrm{RR}$ to understand the transverse and longitudinal dynamics, effectiveness of stochastic cooling system, beam life-time etc. We have also transferred the antiproton beam from the Recycler into the Main Injector and accelerated to $150 \mathrm{GeV}$.

The RR and the MI share the same underground tunnel. The MI acceleration cycles have significant effects on the longitudinal and transverse beam emittance of the stacked beam in the RR. After identification of this problem we have taken several measures to mitigate these effects and to minimize the emittance growth.

Over the past three years we have used different methods of emittance measurement to learn about the longitudinal dynamics at various stages of operation of the Recycler. In this paper, we review the methods used for longitudinal emittance measurements in barrier buckets and discuss their merits and demerits. We also

\footnotetext{
* Work supported by the Universities Research Association, Inc., under contract DE-AC02-76CH03000 with the U.S. Department of Energy.
}

illustrate the use of Monte Carlo methods to estimate the longitudinal emittance in case of a complex beam distribution.

\section{BARRIER BUCKETS AND LONGITUDINAL EMITTANCE}

Use of rf barrier buckets is not new at the Fermilab accelerators. Sinusoidal rf barriers were invented[2] to be used in the antiproton Debuncher and Accumulator rings to produce longitudinal gaps in the stored beam.

A barrier bucket is characterized by barrier pulse shape $V(t)$, pulse duration $T_{1}$ and gap between +ve and -ve pulses $T_{2}$. The general Hamiltonian for an arbitrary barrier rf bucket can be written as [3],

$$
H=-\frac{\eta}{2 \beta^{2} E_{o}}(\Delta E)^{2}-\frac{\int_{o}^{\tau} e V(\tau) d \tau}{T_{0}}
$$

where $\eta$ is the slip-factor of the synchrotron, $\Delta \mathrm{E}$ is energy deviation from synchronous energy $\mathrm{E}_{0}, \mathrm{~T}_{0}$ is the revolution period of the charged particle, $e$ is the electronic charge. The half bucket height is given by,

$\Delta E_{b}=\sqrt{\frac{2 \beta^{2} E_{0}}{|\eta|} \frac{\left|\int_{T_{2} / 2}^{T_{2} / 2+T_{1}} e V(\tau) d \tau\right|}{T_{0}}}$

Here, we assume that the +ve and $-\mathrm{ve}$ pulses are symmetric about the center of the bucket. It is important to note that all physical quantities depend basically on $\int \mathrm{eV}(\tau) \mathrm{d} \tau$ not on the exact shape of the wave form if it had some symmetry about the bucket center.
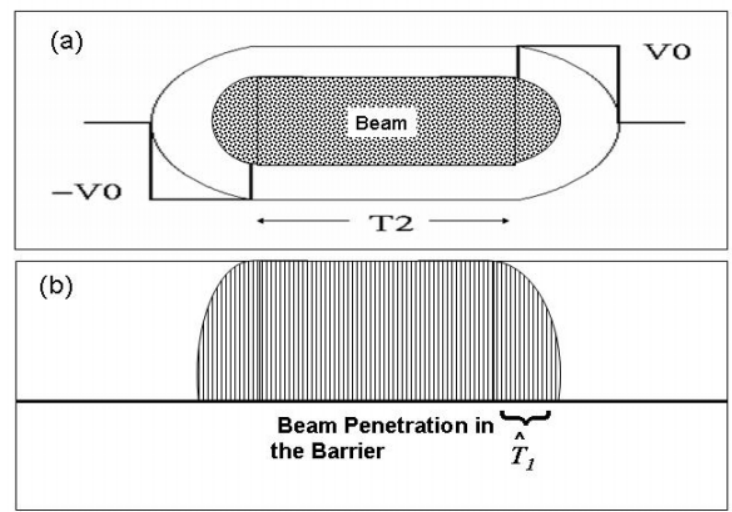

Figure 1: A schematic of RR beam phase-space distribution (a) in a rectangular barrier bucket and, (b) its projection along the time axis. 
The Recycler uses a wide-band (10kHz-100MHz) RF system capable of generating many barrier buckets of any shape[4] . The maximum height of a barrier pulse is set to about $+/-2 \mathrm{kV}$ each. The width of a pulse can be varied. The pbar beam from the antiproton Accumulator will have $2.5 \mathrm{MHz}$ rf structure and that from the Recycler to MI can have $2.5 \mathrm{MHz}$ or $7.5 \mathrm{MHz}$ rf structure. The rf system can also produce $2.5 \mathrm{MHz} / 7.5 \mathrm{MHz}$ rf buckets in between barrier buckets. For a given peak rf voltage a wave form of rectangular shape gives maximum value of $\int \mathrm{V}(\tau) \mathrm{d} \tau$. Hence, we adopted a rectangular barrier bucket. The pulse width is selected to be $\approx 908 \mathrm{nsec}$.

A schematic view of RR barrier waveform with its bucket boundary (an equal Hamiltonian contour defining the bucket) and beam in it is shown in Figure 1. For the beam penetrating in the barrier the beam half height is given by

$$
\Delta \hat{E}_{b}=\sqrt{\frac{2 \beta^{2} E_{0}}{|\eta|} \frac{e V_{b} \hat{T}_{1}}{T_{0}}}
$$

The longitudinal emittance $\varepsilon_{l}$ is given by,

$$
\varepsilon_{l}=2 T_{2} \Delta \hat{E}+\frac{8 \pi|\eta|}{3 \omega_{o} \beta^{2} E_{o} e V_{o}}(\Delta \hat{E})^{3}
$$

where $\omega_{0}=2 \pi / T_{0}$ and $\beta$ is ratio of velocity of the beam to velocity of the light. $\eta=-0.0087, \mathrm{E}_{0}=8.938 \mathrm{GeV}, \mathrm{T}_{0}=$ $11.12 \mu \mathrm{sec}$ for the Recycler.

\section{TECHNIQUES FOR MEASUREMENT OF $\varepsilon_{l}$ and $\Delta \mathbf{E}$}

An accurate measurement of longitudinal emittance of particle beams in any synchrotron is not a trivial task. The knowledge of correct longitudinal emittance is key to the understanding the longitudinal beam dynamics. This inturn helps to improve the proton-antiproton luminosity in the Tevatron. So far, we have used four different measurement techniques in the Recycler. They can be broadly classified into two categories: beam destructive techniques and non-destructive techniques. The destructive techniques are mainly one-time measurements. Their use in a storage ring like the Recycler is very limited. On the other hand, the nondestructive techniques are used as the major beam diagnostics in daily operations. Here, we describe both of these approaches for completeness.

\section{Destructive Techniques}

Two different methods of this type are illustrated here. The first method is based on matching the bucket area to bunch area. After the beam is captured in a barrier bucket the height of the rf pulse is adiabatically reduced while the width is held constant until the beam area fills the bucket. To determine the rf voltage, $\mathrm{V}_{\mathrm{b}}$, where the beam just fills the bucket a gated current integrator (GCI) [5] is used to measure the beam captured between the barriers. A schematic view of this method is shown in Figure 2 (left). Knowing the $V_{b}$ and $T_{1}$ in equations 3 and 4 , the beam energy spread and the beam area are measured. This technique was used in the early detection of the longitudinal emittance growth in the Recycler arising from the MI high energy cycles. Similar measurements can be made if we change the width of the barrier pulse keeping the pulse height constant. For barrier buckets with non-rectangular barrier pulses this method would be less useful.

The second method requires an ability to turn-off the barrier pulses much faster than the synchrotron period of the beam in the barrier bucket. Then the beam energy spread $\Delta \mathrm{E}$, the fractional change in orbiting time $\Delta \mathrm{T}$ and total de-bunching time $\mathrm{T}$ are related according to

$$
\frac{\Delta E}{E}=\frac{\beta^{2}}{\eta} \frac{\Delta T}{T}
$$

In the RR, the synchrotron oscillation period is about of $0.6 \mathrm{sec}$ for a barrier bucket with $\mathrm{T}_{2}=1.6 \mu \mathrm{sec}$ and the beam rf voltage can be turned-off in $<1$ msec. Figure 2 (right inset) illustrates an example of measuring $\Delta \mathrm{E}$ by fast debunching method. The de-bunching time (from bottom to the top) and fractional increase in orbiting time is measured using digitized wall-current monitor data.
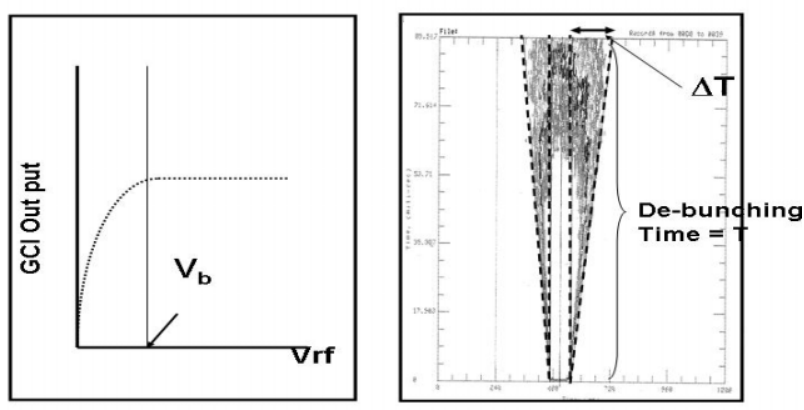

Figure 2: A schematic of the method which adopts varying $\mathrm{rf}$ voltage at a fixed pulse width in RR (left). Typical data from the fast de-bunching method in the RR (right).

\section{Non-destructive Techniques}

We are using two different methods of non-destructive type in the Recycler beam longitudinal emittance studies. The first of these is a variation on the traditional method of measuring the beam profile using a wall current monitor (WCM). A detailed account of use of WCM in longitudinal emittance measurement is given in ref.6. The second method is based on Schottky signal detection.

\section{A. Resistive Wall Current Monitor}

A WCM is a device which measures the image charge that flows along the vacuum chamber following the beam and hence reproduces the longitudinal profile of the beam. 
We have a $4 \mathrm{GHz}$ bandwidth pickup WCM[7] in the Recycler Ring. By knowing the exact location of the barrier pulse and their shapes one measures the beam penetration into the barrier pulse to measure the $\Delta \mathrm{E}$. For a beam confined in a rectangular barrier bucket the beam penetration is symmetric with respect to the center of the bunch. However, any asymmetry in the shape of the pulse or non-zero rf voltage in the gap between +ve and -ve barrier pulse (which might arise due to rf hardware or software systems) will result in an asymmetric beam penetration in the barrier. The WCM then measures different amounts of penetration in the barrier (later we illustrate an example of this type). The WCM data is very sensitive to small distortions in the barrier pulse. In particular, when the longitudinal emittance of the beam is small the WCM allows detection of distortion of the baseline between the barrier pulses.

The Recycler WCM signals are sent to a digitizing scope RDT720. The rf fan-out signals are also processed along with the WCM signals to measure the relative position of the barrier pulse in the ring. A special trigger module is developed to trigger the scope at different times of rf manipulation. Then the data is collected using a software program[8].

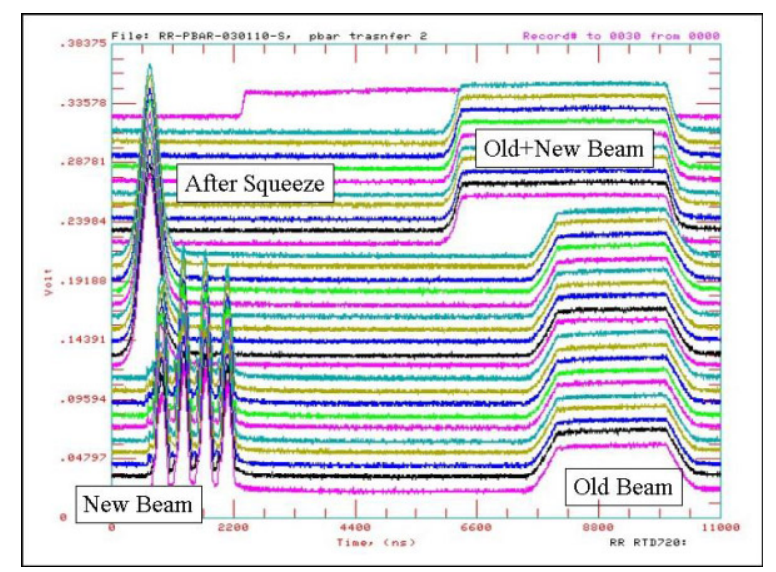

Figure 3: WCM data for antiproton stacking.

Figure 3 illustrates a case of pbar beam stacking in the Recycler. "New Beam" in this figure has $2.5 \mathrm{MHz}$ bunch structure. Knowing the bunch length and the peak rf voltage one can measure the longitudinal emittance of the beam at injection. Measuring the penetration of the beam into the barrier pulses and using equations (3) and (4) the longitudinal emittance of the beam in barrier buckets at various stages are estimated. The errors in the measured emittance mainly come from detector response, estimating the beam penetration into the barrier and the measured rf voltage.

\section{B. Schottky Signals}

Using Schottky signals for beam emittance measurements in a storage ring is an excellent method[9]. For a coasting beam, the energy spread $\Delta E$ and the frequency spread of the Schottky spectrum $\Delta f$ are related according to

$$
\frac{\Delta E}{E}=\frac{\beta^{2}}{\eta} \frac{\Delta f}{f}
$$

where $f=n f_{0}, f_{0}$ is the revolution frequency of the synchronous particles and $n$ is the harmonic number. Measuring the maximum energy spread using a Schottky detector resonating at a reasonable harmonic number $(\mathrm{n} \sim 100)$ and a spectrum analyzer is straight forward.

In case of a sinusoidal bunched beam, the beam particles execute synchrotron oscillations. The time of passage of a particle in front of the detector is modulated according to synchrotron oscillation amplitude. Thus, the time coordinate $t \rightarrow t+\tau \sin (\Omega t+\phi)$ as compared to a coasting beam. The quantities $\tau, \Omega$, and $\phi$ are synchrotron amplitude, frequency and phase, respectively. Then the beam current for a single particle in frequency domain will take the form

$i(t)=e f_{0}+2 e f_{0} \sum_{n=1}^{\infty} \sum_{k=-\infty}^{k=\infty} J_{k}\left(n \omega_{0} \tau\right) \cos \left[\left(n \omega_{0}+k \Omega\right) t+k \phi\right]$

where $J_{k}$ is the Bessel function of order $k$. Hence, in the case of bunched beam with some energy spread, each revolution frequency band at frequency $n f_{0}$ (as seen in the coasting beam) is replaced by a central line with an infinite number of satellites. Further, the satellites of order $\mathrm{k}$ with different values of $n$ are correlated which gives rise to coherence. This feature of the bunched beam Schottky spectrum makes it more complicated to interpret for longitudinal emittance measurements. Fortunately, for sufficiently large $n$, (at high frequency), the equation (6) is an excellent approximation for all types of beams.
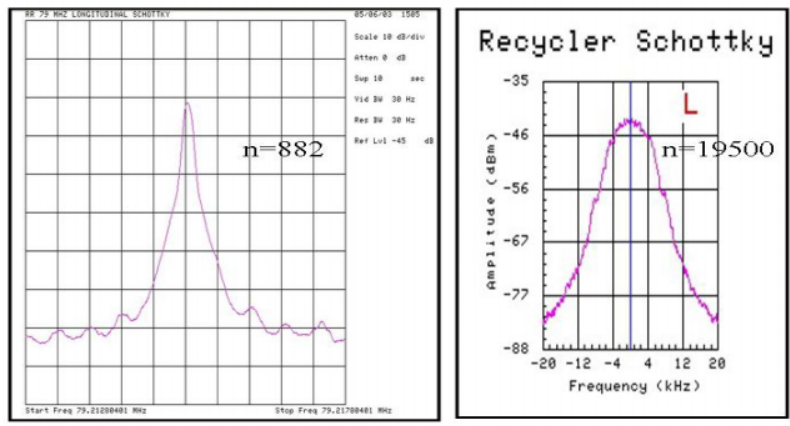

Figure 4: Typical Schottky spectra taken with two detectors. Left $(n=882)$ and right $(n=19500)$.

The beam bunched in barrier buckets can be described in terms of a sum over harmonics of the synchrotron frequency, but the synchrotron frequency spread is so big that the signal from a beam bunched in barrier is very similar to a truly coasting beam.

At the Recycler, we have used three Schottky detectors with frequencies $79 \mathrm{MHz}, 1.5 \mathrm{GHz}$ and $1.75 \mathrm{GHz}$. They have $n=882,16700,19500$, respectively. In Figure 4, we illustrate a typical longitudinal Schottky spectrum from two detectors with widely different harmonic 
numbers. The data are taken for the same beam in a barrier bucket. The data shown on left are obtained with $79 \mathrm{MHz}$ Schottky detector and show in addition to the Schottky signal a prominent coherent peak at revolution harmonic. Hence, unambiguous determination of $\Delta E$ is difficult. The figure on the right hand side shows data with the $1.75 \mathrm{GHz}$ Schottky detector and does not show coherent peak. We find that the maximum energy spread is about $5.6 \mathrm{MeV}$ for the case illustrated here.

It is important to note that the measured $\Delta E$ using Schottky spectrum is independent of rf voltage wave form if spectrum does not show any coherent peak. However, the Schottky technique does not distinguish between captured beam or an un-captured beam. It can be gated to measure different parts of the beam.

\section{Comparison between WCM and Schottky Methods}

We have carried out a number of experiments to measure longitudinal emittance of the beam in Recycler Ring using Schottky signals and by using WCM. Here we illustrate two examples from these measurements

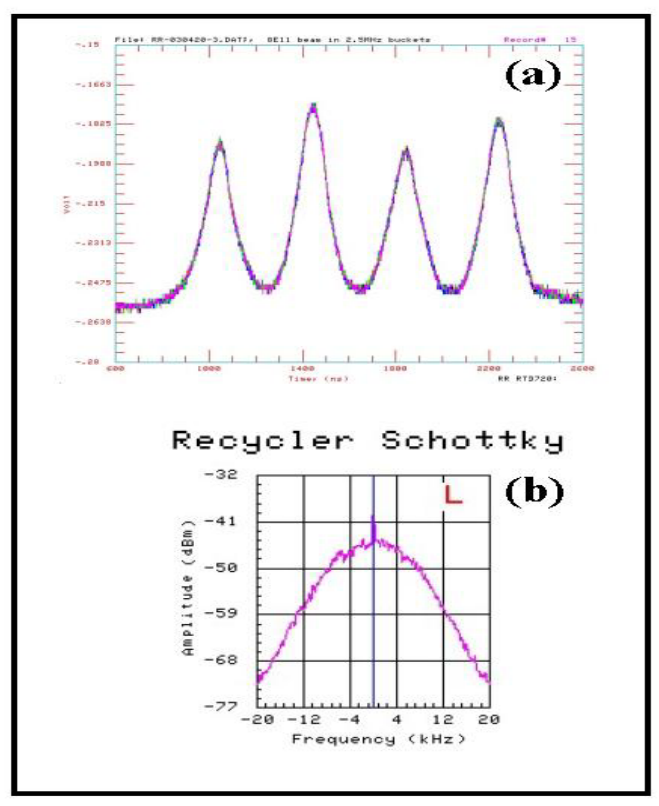

Figure 5: (a) WCM data for bunched beam in the Recycler, (b) $1.75 \mathrm{GHz}$ Schottky spectrum for the same beam.

Fig. 5 shows data from WCM and $1.75 \mathrm{GHz}$ Schottky detector for beam in four $2.5 \mathrm{MHz}$ buckets. The $2.5 \mathrm{MHz}$ rf voltage was about $2 \mathrm{kV}$ and average bunch length $(90 \%$ by area) is about 260 nsec. We find that the measured emittance for assumed elliptical distribution to be $20 \mathrm{eVs}$ and $2 \Delta \mathrm{E} \approx 12 \mathrm{MeV}(<20 \%)$. For the same beam the Schottky spectrum gives about $2 \Delta \mathrm{E} \approx 9 \mathrm{MeV}$.

Data taken for the beam in a typical barrier bucket are shown in Figure 6 . In this case $T_{2}=1.6 \mu$ sec. The measured $\varepsilon_{l} \approx 14 \mathrm{eVs}$ and $\Delta \mathrm{E} \approx 8 \mathrm{MeV}$ from the WCM measurements can be compared to $\varepsilon_{l} \approx 9 \mathrm{eVs}$ and $\Delta \mathrm{E} \approx 5.6 \mathrm{MeV}$ from Schottky measurements.

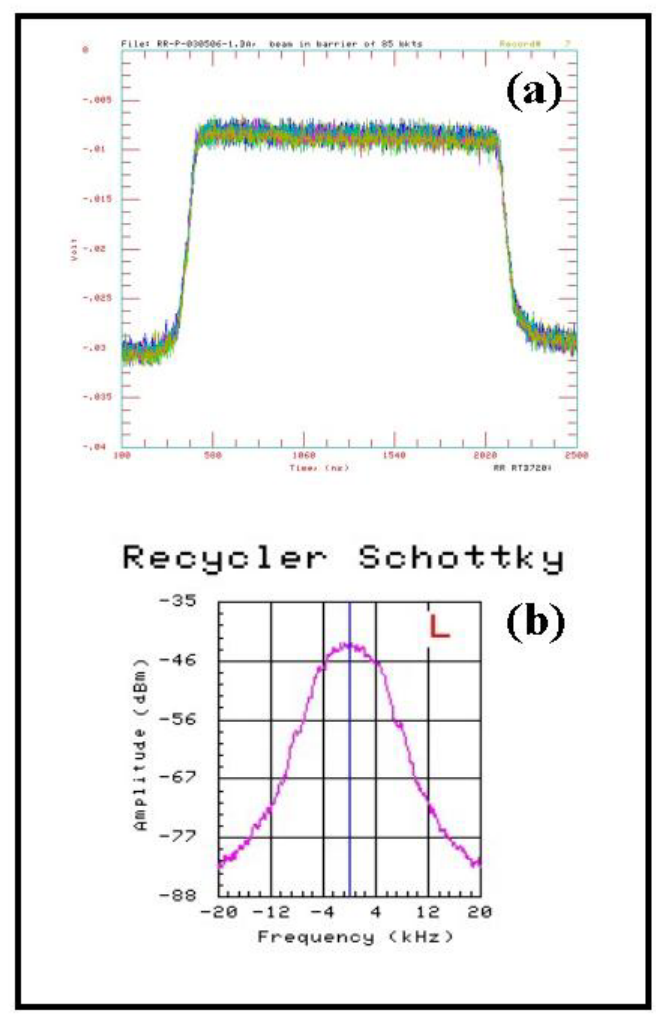

Figure 6: (a) WCM data for the beam in a rectangular barrier bucket, (b) $1.75 \mathrm{GHz}$ Schottky spectrum for the same beam. $\mathrm{T}_{1}=908 \mathrm{nsec}, \mathrm{T}_{2}=1.6 \mathrm{msec}$ and $\mathrm{V}_{0}=2 \mathrm{kV}$.

\section{Some Special Cases}

The perfect rectangular barrier pulse discussed above is not reality in the Recycler. Typically, a rectangular barrier pulse in the Recycler will be have rounded edges. In between barrier pulses there may be a small slope of a few percent of the total pulse amplitude. Besides, as one adds a number of barrier pulses around the accelerator ring the adjacent barrier pulses are found to be distorted at a few percent level. This phenomenon makes the measurement of longitudinal emittance more difficult.

Figure 7 illustrates a case with non-symmetric Vrf between barrier pulses. Figure 7(a) shows a WCM data for cooled pbar beam. We have made an attempt to simulate the WCM data using a multi-particle beam dynamics code (a Monte Carlo program), ESME[10]. In our model we have assumed a damping oscillating rf wave with a $\operatorname{Vrf}(\max )=2.5 \%$ of $2 \mathrm{kV}$ between the barrier pulses which starts at the negative barrier pulse. This method predicts the longitudinal emittance to be about $51 \mathrm{eVs}$ and $\Delta \mathrm{E}_{\max } \approx 8 \mathrm{MeV}$. The energy spread estimated here can be compared with the Schottky signal method of 6.4MeV ( at $-10 \mathrm{~dB})$. 


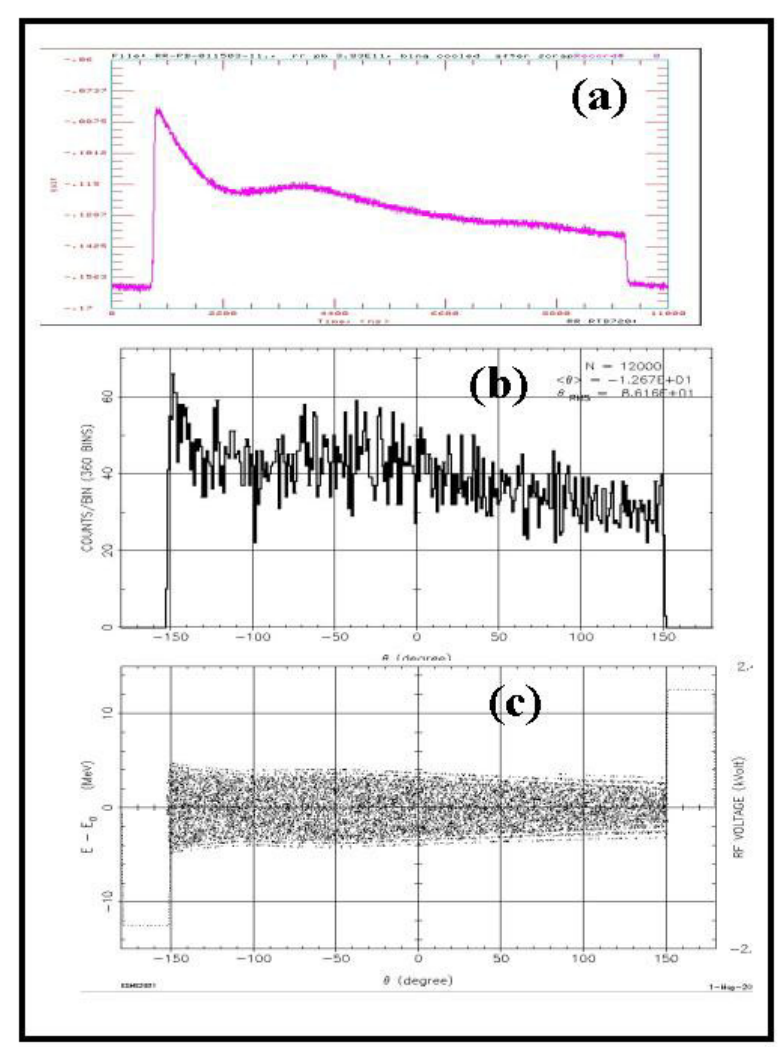

Figure 7: (a) WCM data for the cooled pbar beam in a rectangular barrier bucket with non-zero and nonsymmetric Vrf in between the barrier pulses. (b) ESME multiparticle beam dynamics simulation for the distribution shown in (a). The $(\Delta \mathrm{E}, \Delta \theta)$-phase space distribution of beam particles as predicted from the simulation.

In summary we have measured the longitudinal emittance of the beam in the Recycler Ring by using four different techniques, two destructive and two nondestructive techniques. The WCM and Schottky spectrum methods are very promising, and are suitable for future applications in Recycler during routine operation. A more detailed understanding of the measurement errors is the subject of future effort.
The authors would like to thank D. Wildman and J. MacLachlan for many useful discussions, A. Cadorna, B. Fellenz (gated current integrator), Brian Chase (for LLRF settings), Ming-Jen Yang (for console application program to extract the digitized WCM data), Joe Dey and J. Reid (for their help in HLRF), M. Hu and D. Broemmelsiek (Schottky Detector settings) and Craig McClure (trigger circuit for RTD720 scope), and Operation group for their help during the course of this work.

\section{REFERENCES}

[1] G. Jackson, "The Fermilab Recycler Ring Technical Design Report" FERMILAB-TM-1991 (1996).

[2] G.E. Griffin et. al, IEEE, Trans. Nucl. Sci., NS-30, 3502 (1983).

[3] S.Y. Lee and K. Y. Ng, Phys. Rev. E, Vol. 55, 5992 (1997).

[4] J.E. Dey and D.W. Wildman, IEEE, Proc. the 1999 Part. Accel. Conf. New York, NY, 869, 1999).

[5] A. Cadorna et. al., "Gated Current Integrator for the Beam in the RR Barrier Buckets," this proceedings.

[6] R.C. Webber, AIP Conference Proceedings No. 212, page 85-126, edited by E.R Beadle and V.J. Castillo, Accelerator Instrumentation Upton, NY, 1989.

[7] Brian Fellenz and J. Crisp (private communications).

[8] Ming-Jen Yang, A console applications program (unpublished).

[9] D. Boussard, CERN SPS/86-11(ARF), Geneva 1986.

[10] J. MacLachlan, ESME modified to handle beam dynamics in barrier buckets (1996), (private communications 2001). 